\title{
Validation of LBM based on BGK on Poiseuille's Flow and Vortex Street in a Channel Flow
}

\author{
Alankar Agarwal, Akshay Prakash
}

\begin{abstract}
The aim of research is to simulate a large scale bioreactor using Lattice Boltzmann method (LBM). Design and scale up a bioreactor is a highly complex process since it involves various physical phenomena occurring together. Computational Fluid Dynamics (CFD) has been developed over the years to simulate complex phenomenon occurring in a bioreactor and can make up for the shortcomings associated with traditional scale-up methods. It allows the simulation of all the relevant physics like species and thermal transport along with millions of bubbles. Due to various recent advances in numerical techniques and computer hardware (particularly the Graphical Processing Units-GPUs, aimed at number crunching applications), the simulation of an industrial scale reactor is now possible in reasonable time. While conventional pressure based solvers, such as the SIMPLE algorithm and its variants, do not scale well with large number of processors and may not utilize the GPUs cores effectively, Lattice Boltzmann Methods are highly parallelizable and scale almost linearly with number of cores. In this paper we present the development and validation of a basic $L B M$ code which will be used for the bioreactor simulation. The Bhatnagar-GrossKrook (BGK) model is used and is validated by theoretically computed parameters for Poiseuille's flow and with experimentally determined parameters for Vortex Street in a channel flow. Velocity profile, Strouhal number and vortex distance are compared. Reynolds numbers $(\mathrm{Re})$ of $30,60,120$, and 140 have been considered.
\end{abstract}

Keywords-Lattice Boltzmann Method, Computational Fluid Dynamic, Bhatnagar-Gross-Krook, Von karman vortex street

\section{Introduction}

Recently, Lattice Boltzmann Method (LBM) has emerged as a valuable simulation technique for solving fluid flow problems and modeling physics in fluids. It evolves from the continuous Boltzmann transport equation. The main advantage of LBM is that it computes a flow field through localized operations of streaming and collision. These increase the parallelizability of the algorithm. Also, the particle based bounce-back boundary conditions decrease the complexity, but then only a stair stepped boundary is possible. LBM has been shown to predict the flow parameters accurately for turbulence, magneto hydrodynamic flows across complex geometries to multiphase flows with phase change [1].

This paper presents, simulation of Poiseuille' flow and flow past a square cylinder in a channel using LBM. This flow is laminar at very low Reynolds numbers (Re), and as

Alankar Agarwal, Akshay Prakash

Dept. of Mechanical Engineering - Indian Institute of Technology Jodhpur India the Reynolds number goes on increasing the flow separates from the stagnation points but remain laminar and steady up to $\mathrm{Re}=50$. Beyond this Reynolds number unsteadiness develops, and flow become periodic [2].

Furthermore, on the basis of these results, we would simulate the numerous parameters to scale-up large scale stirred tank reactor $\left(\sim 100 \mathrm{~m}^{3}\right)$ for the production of Escherichia Coli (E.coli). Stirred tank reactors are among the most popularly used reactor in chemical and pharmaceutical industries. E.coli is a prokaryote commonly found in the lower intestine of human and warm blooded organisms. The optimal growth of E.coli is dependent upon $\mathrm{pH}$, temperature, dissolved oxygen concentration and nutrient availability in the medium [3]. It is necessary to scale-up numerous parameters of stirred tank reactor, which include bubble interaction, multiphase flows, bubble diameter, impeller speed, biogas volume fraction, turbulence kinetic energy and shear strain rate, etc to achieve the optimal growth of E.coli [4].

\section{Methods}

Lattice Boltzmann method is relatively a new method in the field of Computational Fluid Dynamics (CFD). It can be used to simulate incompressible as well as compressible flows with heat and species transfer. LBM time step translates to a very small physical interval. Hence it is also called mesoscopic model. LBM is also efficient for solving complex fluid flow problems such as complex boundary conditions, miscible and immiscible fluid and thermal effects, when compared with conventional CFD techniques [5].

Conventional CFD techniques based on continuum theory, solve macroscopic fluid dynamics equations by using various numerical partial differential discretization method (such as finite difference method (FDM), finite volume method (FVM) and finite element method (FEM)), whereas LBM solves problem at mesoscopic scale with the real- valued particle distribution functions $f(x, c, t)$, it depends on the position vector $x$, the velocity vector $c$ and time $t$. In each simulation time step, values of particle distribution function are updated through two sequential stages: streaming and collision. In the streaming stage, particle at position $x$ after time $d t$ move to $x+d x$ in the direction of its velocity. The collision stage involves only those, real valued particle distribution function arriving at each node although in some other cases (such as multicomponent and multiphase flows) particle distribution function at other nodes may also influence the collision step [6].

Currently, the most popular LBM method used is latticeBGK model (LBGK). It has been applied in variety of complex flows. LBGK model is about relaxing nonequilibrium velocity distributions to equilibrium value $\boldsymbol{\tau}$. Since the value of $\boldsymbol{\tau}$ is same for all the conservation 
equations (mass and momentum) it is called a single relaxation model. In this model, collision process is represented to be a single-time relaxation to the local equilibrium distribution [7]. The evolution of lattice Boltzmann equation from the continuous Boltzmann-BGK model is given by [8]:

$$
f_{j}\left(\vec{x}_{j}+\vec{c}_{j} d t, t+d t\right)-f_{j}\left(\vec{x}_{j}, t\right)=-\frac{1}{\tau}\left(f_{j}-f_{j}^{e q)}\right.
$$

where $d t$ is increment in time, $f_{j}$ is the particle velocity distribution along the $j$ direction, $\boldsymbol{\tau}$ is the dimensionless relaxation time parameter towards local equilibrium and $f_{j}^{e q}$ is the equilibrium distribution function.

In the LBGK model, the structure of $\mathrm{D} m \mathrm{Q} b$ model for $m$ (microscopic) velocity models in $b$ directions are the most popular ones [6]. All the numerical simulation in this paper considered two-dimensional square lattice structure. D2Q9 has been broadly and effectively utilized for simulation of two-dimensional fluid flow problem. This model also referred as nine-velocity square lattice model. Fig.1 shows the representation of D2Q9 lattice arrangement.

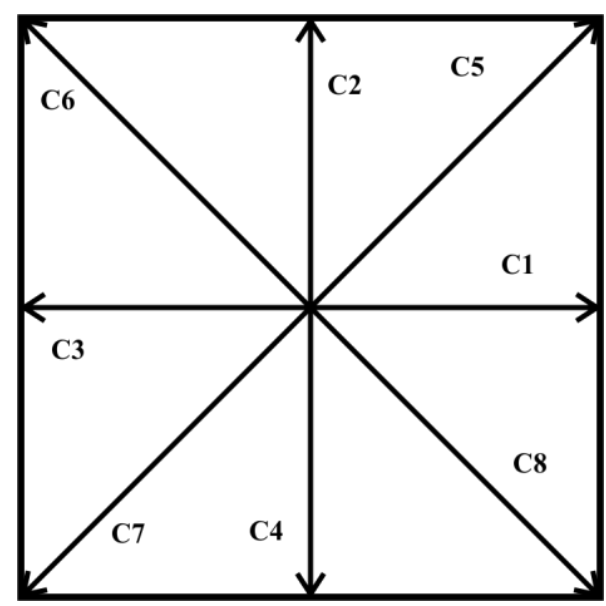

Figure 1. Structure of two dimensional nine velocity square lattice (D2Q9) model.

In the $\mathrm{D} 2 \mathrm{Q} 9$ model $c_{j}$, denotes the discrete velocity set and can be expressed as [7]:

$$
c_{j}=\left\{\begin{array}{c}
(0,0), j=0 \\
e\left(\cos \theta_{j}, \sin \theta_{j}\right), \theta_{j}=(j-1) \frac{\pi}{2} \quad, j=1,2,3,4 \\
\sqrt{2}\left(\cos \theta_{j}, \sin \theta_{j}\right), \theta_{j}=(j-1) \frac{\pi}{4}, j=5,6,7,8
\end{array}\right.
$$

where $e=d x / d t$. The equilibrium distribution function for the D2Q9 model is given by [5]:

$$
f_{j}^{e q}=\rho w_{j}\left(1+\frac{3}{e^{2}} \vec{c}_{j} \cdot \vec{u}+\frac{9}{2 e^{4}}\left(\vec{c}_{j} \cdot \vec{u}\right)^{2}-\frac{3}{2 e^{2}} u \cdot u\right)
$$

where $u=\left(u_{x}, u_{y}\right)$ be the macroscopic velocity, $w$ be the weighting factor expressed as:

$$
w_{j}=\left\{\begin{array}{c}
\frac{4}{9}, j=0 \\
\frac{1}{9}, j=1,2,3,4 \\
\frac{1}{36}, j=5,6,7,8
\end{array}\right.
$$

Eq.1 is referred as the Lattice Boltzmann Equation (model) for BGK model. It consist of following two steps, which updated after every time step $d t$.

Collision step:

$$
f_{j}\left(\vec{x}_{j}, t+d t\right)=f_{j}\left(\vec{x}_{j}, t\right)-\frac{1}{\tau}\left(f_{j}\left(\vec{x}_{j}, t\right)-f_{j}^{e q}\left(\vec{x}_{j}, t\right)\right)
$$

Streaming step:

$$
f_{j}\left(\vec{x}_{j}+\vec{c}_{j} d t, t+d t\right)=f_{j}\left(\vec{x}_{j}, t+d t\right)
$$

The relaxation time parameter $\boldsymbol{\tau}$ is related to the viscosity that fixes the rate of approach to equilibrium given by [5]:

$$
v=\frac{2 \tau-1}{6} \frac{(d x)^{2}}{d t}
$$

The macroscopic property such as density per node and momentum density are evaluated from the real-valued particle distribution function by [7]:

$$
\sum_{j=0}^{8} f_{j}=\rho, \sum_{j=0}^{8} f_{j} c_{j}=\rho u
$$

This density and momentum density satisfy the conventional pressure based equations (i.e. Navier-Stokes equations) in the low Mach number limit explained by using the Chapman-Enskog expansion [9].

Fig .2 shows flow chart of algorithm involved in LBGK model [10].

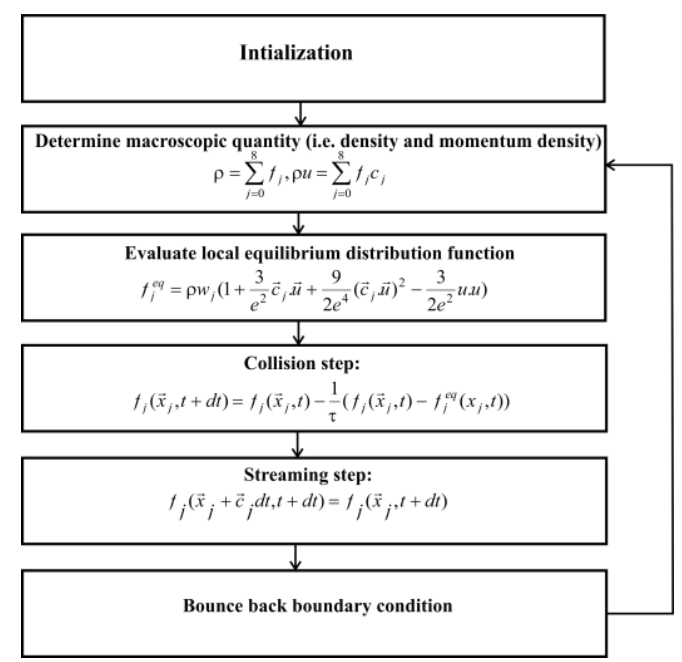

Figure 2. Flowchart of algorithm involved in LBGK model [10] 


\section{Problem Description and Formulation}

We study the flow past a square cylinder with diameter $D$, that is residing centrally inside a plane channel having height $H$ with blockage ratio $B=D / H=1 / 8$ and at a fixed channel length of $L / D=50$. An inflow length of $l=L / 4$ or $12.5 D$ has been chosen [11]. Fig.3 shows the schematic representation of flow past a square cylinder in a channel.

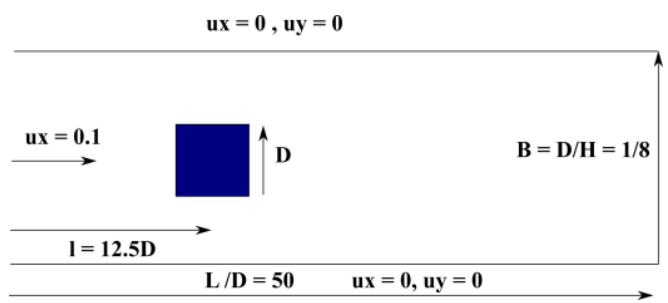

Figure 3. Schematic representation of flow past a square cylinder placed centrally inside a channel.

The LBM simulation were performed at moderate Reynolds number $\operatorname{Re}=\mathrm{u}_{\mathrm{x}} \mathrm{D} / \mathbf{v}$, where $u_{x}$ is the inflow velocity, $\mathrm{D}$ is the diameter of square cylinder and $\mathbf{v}$ is the kinematic viscosity of water. The physical inflow velocity $u_{x}$ is 0.014 $\mathrm{m} / \mathrm{s}$.

The parameters involved in simulation for Poiseuille's flow and Vortex Street in a channel flow is given in table.1 and table. 2 respectively.

TABLE I. SimULATION PARAMETERS FOR POISEUILLE's Flow

\begin{tabular}{|l|l|c|}
\hline \multicolumn{1}{|c|}{ S. No. } & \multicolumn{1}{|c|}{ Parameters } & $\begin{array}{c}\text { Values in lattice } \\
\text { unit }\end{array}$ \\
\hline 1 & No. of grid points in x-direction & 250 \\
\hline 2 & No. of Grid points in y-direction & 40 \\
\hline 3 & Inlet velocity & 0.1 \\
\hline 4 & Relaxation time $(\tau)$ & 0.6 \\
\hline
\end{tabular}

TABLE II. Simulation PARAMETERS FOR FlOW PAST A SQUARE CYLINDER

\begin{tabular}{|l|l|c|c|c|}
\hline S. No. & $\begin{array}{c}\text { Reynolds } \\
\text { number }\end{array}$ & $\begin{array}{c}\text { Diameter } \\
\text { (physical } \\
\text { unit)(m) }\end{array}$ & $\begin{array}{c}\text { Diameter } \\
\text { (lattice } \\
\text { unit })\end{array}$ & Grid Size \\
\hline 1 & 30 & 0.0336 & 10 & $500 \times 80$ \\
\hline 2 & 60 & 0.0672 & 20 & $1000 \times 160$ \\
\hline 3 & 120 & 0.1344 & 40 & $2000 \times 320$ \\
\hline 4 & 140 & 0.1568 & 48 & $2400 \times 384$ \\
\hline 5 & 160 & 0.1792 & 54 & $2700 \times 432$ \\
\hline
\end{tabular}

\section{Boundary Conditions}

Boundary conditions based on non-equilibrium bounce back condition given by Zuo and He are used [12].

\section{A. Bottom and Top Wall of Channel}

The boundary of the bottom wall of channel is aligned with x-direction. For example, take the case of a bottom node, where $f_{4}, f_{7}, f_{8}$ pointing into the wall. After the streaming process, value of $f_{0}, f_{1}, f_{3}, f_{4}, f_{7}$, and $f_{8}$ particle distribution function are known. As, no slip boundary condition on the wall, provides $u_{x}=u_{y}=0$, Eq. (8) has used to evaluate $f_{2}, f_{5}, f_{6}$, and $\boldsymbol{\rho}$ which can be put into the form:

$$
\begin{aligned}
& f_{2}+f_{5}+f_{6}+\left(f_{0}+f_{1}+f_{3}+f_{4}+f_{7}+f_{8}\right)=\rho \\
& f_{5}-f_{6}+\left(f_{1}-f_{3}-f_{7}+f_{8}\right)=\rho u_{x} \\
& f_{2}+f_{5}+f_{6}+\left(f_{4}+f_{7}+f_{8}\right)=\rho u_{y}
\end{aligned}
$$

Solving Eqs. (9) and (10) gives:

$$
\rho=\frac{1}{1-u_{y}}\left[f_{0}+f_{1}+f_{3}+2\left(f_{4}+f_{7}+f_{8}\right)\right]
$$

However, the value of distribution functions $f_{2}, f_{5}$, and $f_{6}$ remains unchanged. Thus, bounce back scheme is used to determine $f_{2}, f_{5}$, and $f_{6}$ given by [12]:

$$
\begin{aligned}
& f_{2}-f_{2}^{e q}=f_{4}-f_{4}^{e q} \\
& f_{2}=f_{4}+\frac{2}{3} \rho u_{y} \\
& f_{5}=\frac{1}{6} \rho u_{y}+f_{7}-\frac{1}{2}\left(f_{1}-f_{3}\right)+\frac{1}{2} \rho u_{x} \\
& f_{6}=\frac{1}{6} \rho u_{y}+f_{8}+\frac{1}{2}\left(f_{1}-f_{3}\right)-\frac{1}{2} \rho u_{x}
\end{aligned}
$$

Using Eq. (8) similar procedure can repeat to determine $f_{4}, f_{7}, f_{8}$ particle distribution function for the top boundary wall.

\section{B. Inlet and Outlet}

Using pressure boundary condition at the inlet, along ydirection. In a plane channel flow $u_{y}=0$ at the inlet. After completion of streaming process, $f_{2}, f_{3}, f_{4}, f_{6}$, and $f_{7}$ are known. We need to evaluate $u_{x}$ and $f_{1}, f_{5}$, and $f_{8}$ from Eq. (8) given by:

$$
\begin{aligned}
& f_{0}+f_{1}+f_{2}+f_{3}+f_{4}+f_{5}+f_{6}+f_{7}+f_{8}=\rho \\
& \rho u_{x}=f_{1}+f_{5}+f_{8}-\left(f_{3}+f_{6}+f_{7}\right) \\
& f_{2}-f_{4}+f_{5}-f_{7}+f_{6}-f_{8}=u_{y}=0
\end{aligned}
$$

Solving Eqs. (14) and (15) gives:

$$
u_{x}=1-\frac{\left\lfloor f_{0}+f_{2}+f_{4}+2\left(f_{3}+f_{6}+f_{7)}\right\rfloor\right.}{\rho}
$$

From the bounce back scheme we have to find out the value of $f_{1}, f_{5}$, and $f_{8}$. 


$$
\begin{aligned}
& f_{1}-f_{1}^{e q}=f_{3}-f_{3}^{e q} \\
& f_{1}=f_{3}+\frac{2}{3} \rho u_{x} \\
& f_{5}=\frac{1}{6} \rho u_{x}+f_{7}-\frac{1}{2}\left(f_{2}-f_{4}\right) \\
& f_{5}=\frac{1}{6} \rho u_{x}+f_{6}+\frac{1}{2}\left(f_{2}-f_{4}\right)
\end{aligned}
$$

A similar procedure can be applied for the outlet.

\section{Corner Nodes}

The corner node normal to inlet/outlet and boundary wall requires some special treatment. As an example, take the case of bottom node at the inlet. After the streaming step, $f_{3}$, $f_{4}$, and $f_{7}$ are known, density $\boldsymbol{\rho}$ is specified, and from no slip $u_{x}=u_{y}=0$. The bounce back rule for non-equilibrium part of particle distribution function, is used to determine $f_{1}, f_{2}, f_{5}, f_{6}$, and $f_{8}$ given by:

$$
\begin{aligned}
& f_{1}=\left(f_{1}^{e q}-f_{3}^{e q}\right)+f_{3}=f_{3} \\
& f_{2}=\left(f_{2}^{e q}-f_{4}^{e q}\right)+f_{4}=f_{4}
\end{aligned}
$$

Inserting values of $f_{1}$ and $f_{2}$ from Eq. (9) in Eqs. (15), (16) gives:

$$
\begin{aligned}
& f_{5}=f_{7} \\
& f_{6}=f_{8}=\frac{1}{2}\left[\left(f_{0}+f_{1}+f_{2}+f_{3}+f_{4}+f_{5}+f_{7}\right)-\rho\right]
\end{aligned}
$$

\section{Obstacle Walls}

A half way bounce back scheme is used as the boundary condition on the obstacle walls given by:

$$
f_{2}=f_{4}, f_{3}=f_{1}, f_{5}=f_{7}, f_{6}=f_{8}
$$

\section{v. Results and Discussion}

The simulation was carried out using LBGK at different Reynolds numbers with fixed blockage ratio. For all cases, simulations were performed for 50,000 iterations (1200 sec in physical space). Then, LBGK code is validated theoretically computed parameters for Poiseuille's flow and experimentally determined parameters for flow past a square cylinder.

\section{A. Poiseuille's Flow}

To validate, LBGK model, we compare our simulation results for Poiseuille's flow with its analytical solution. Eq. (22) represent the differential equation of plane Poiseuille's flow given by [13]:

$$
\frac{d p}{d x}=\mu \frac{d^{2} u}{d y^{2}}
$$

Solving Eq. (22) analytically gives:

$$
u(x, y)=\frac{\Delta p}{2 * v^{*} \rho^{*} L} y(H-y)
$$

where, $\Delta p$ is calculated from the Darcy-Weisbach equation [14],

$$
\Delta p=32 * v^{*} \rho * L^{*} u_{i n} / H^{2}
$$

where, $L=$ is the channel length, $\mathrm{H}$ is channel width and $u_{\text {in }}$ is the inlet velocity, $\boldsymbol{\rho}$ is the density of water, and $\mathbf{v}$ is the kinematic viscosity of water. Fig.4 shows the comparison of velocity profile in a plane Poiseuille's flow in y-direction between LBGK model and analytical solution.

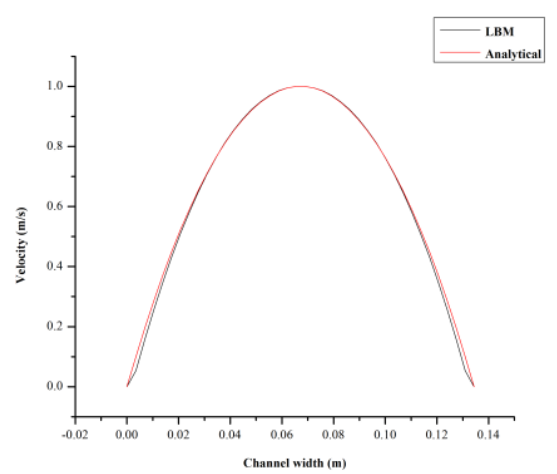

Figure 4. Comparison of velocity profile in a plane Poiseuille's flow between LBGK model and analytical solution.

\section{B. Flow Past a Square Cylinder}

The flow past a square cylinder were investigated at moderate Reynolds numbers $(\mathrm{Re})$. All the simulation were carried out at fixed blockage ration $B=D / H=1 / 8$, using SRT/ LBGK D2Q9 model. Fig.5 shows the velocity contour around square cylinder at different Reynolds numbers (Re).
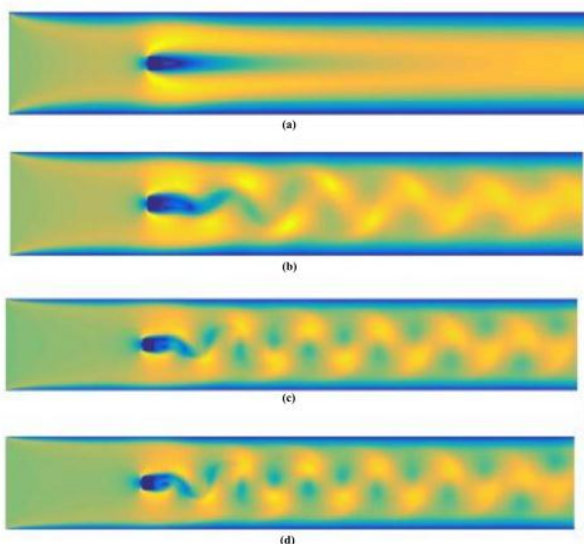

Figure 5. Velocity contour around square cylinder at $\operatorname{Re}=$ (a) 30, (b) 60, (c) 120 , and (d) 140 .

To validate the simulation results for the vortex shedding pattern, we compared the distance between two vortices and Strouhal number at $\mathrm{Re}=140$, with its experimentally determined value evaluated earlier by researchers $[15,16]$.

The distance between two vortices is $1.05 \mathrm{~cm}$ through our simulation result and $1.16 \mathrm{~cm}$ from available experimental data at $\operatorname{Re}=140$ [15].

Strouhal number is a dimensional less quantity used to describe oscillating flow mechanism given by [6]: 


$$
S_{t}=\frac{f D}{u}
$$

where, $f$ is the vortex shedding frequency, $\mathrm{D}$ is the diameter of square cylinder and $u$ is the flow velocity. To calculate the vortex shedding frequency, FFT analysis of the time series data of the flow velocity at different location for $\operatorname{Re}=140$ was performed. Fig. 6 shows the time series graph of the flow velocity at different locations for $\mathrm{Re}=140$ and its FFT analysis.
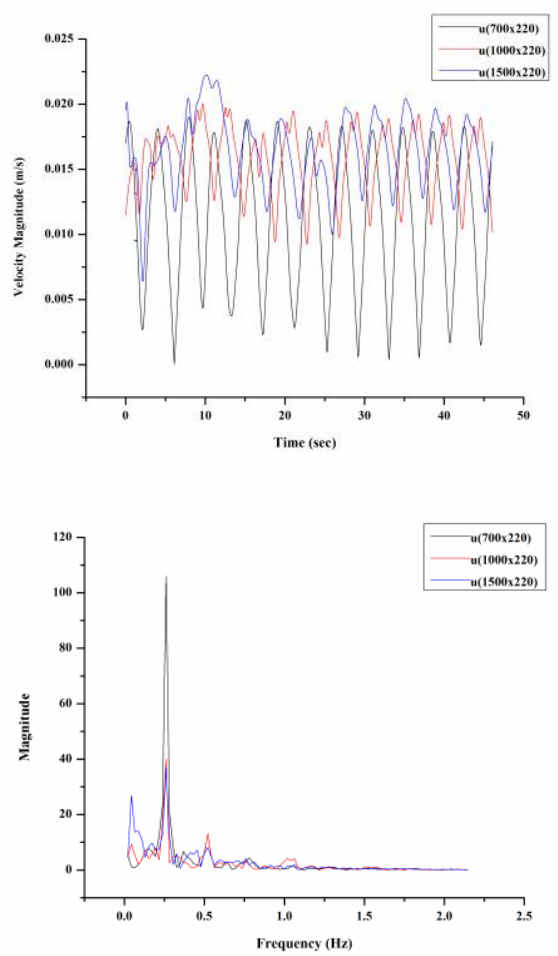

Figure 6. Time series graph of flow velocity at different locations and its FFT analysis for $\operatorname{Re}=140$.

The value of Strouhal number $(\mathrm{St})$ at $\mathrm{Re}=140$ estimated by our simulation is 0.186 and 0.182 from available experimental data provided by Williamson [15].

\section{vi. Conclusion}

In this paper, simulations are carried out for the plane Poiseuille's flow and flow past a square cylinder using LBGK model of Lattice Boltzmann method. Initially, the basic LBGK code is developed for Poiseuille's flow and then validated with its analytical solution. The simulation results give the parabolic curve for velocity profile in the ydirection, which is consistent with its analytical solution. Furthermore, LBGK code is used to simulate the flow past a square cylinder, and study the effect of Reynolds number (Re) on the flow behavior. Results show that flow starts separating from the stagnation point but remain laminar at $\operatorname{Re}=30$, but as the Reynolds number goes on increasing unsteadiness develops and flow become periodic. The distance between two vortices at $\operatorname{Re}=140$ are then measured both for the simulation result and for available experimental data. The simulation shows almost identical results with the experimental result. The Strouhal number for $\operatorname{Re}=140$ also calculated to compare it with the experimental value. The simulated value of Strouhal number for $\operatorname{Re}=140$ found very much close to it experimental value. Basing on the work presented here, we continue to develop the code and model for the simulation to scale-up the various parameters involved in stirred tank bioreactor such as multiphase flows, bubble interaction, thermal transport for E.Coli production using LBM.

\section{References}

[1] W. Regulski, and J. Szumbarski, "Numerical simulation of confined flows past obstacles-the compartive study of Lattice Boltzmann and Spectral Element Method," Archives of Mechanics, vol. 64.4, pp. 423-456, 2012

[2] P. D. Arumuga, V. S. K. Gundavarapu, and A. K. Dass, "Numerical simulation of viscous flow over a cylinder using lattice boltzmann method," ISRN Mathematical Physics, 2012.

[3] R. Murthasan, "Bioreactors," Engineeing Biotechnology: Gateway Project.

[4] R. Sungkorn, J. J. Derksen, and J. G. khinast, "Modeling of turbulent gas-liquid bubbly flows using stochastic Lagrangian model and lattice-Boltzmann scheme," Chemical engineering science, vol. 18.4, pp. 1235-1246, 2014

[5] R. Zhang, and H. Chen "Lattice Boltzmann method for simulations of liquid-vapor thermal flows," ," Physical Review E 67, vol. 6, pp. 066711,2003

[6] J. Yojina, W. Ngamsaad, N. Nuttavut, D. Triampo, Y. Lenbury, P. Kanthang, S. Sriyab, and W. Triampo, "Investigating flow patterns in a channel with complex obstacles using the lattice Boltzmann method," Journal of mechanical science and technology 24, vol. 10, pp. 2025-2034, 2010.

[7] P. L. Bhatnagar, P. Lal, E. P. Gross, and M. Krook, "A model for collision processes in gases. I. Small amplitude processed in charged and neutral one-component systems," Physical Review, vol. 94.3, pp. $511,1954$.

[8] X. He, and L. S. Luo, "Theory of the Lattice Boltzmann method: From the Boltzmann equation to the lattice Boltzmann equation," Physical Review, vol. E 56.6, pp. 6811, 1997.

[9] X. He, and L. S. Luo, "Lattice Boltzmann model for the incompressible Navier-Stokes equation," Journal of statistical Physics, vol. 88.3-4, pp. 927-944, 1997.

[10] Thyholdt, K. Clausen, Lattice Boltzmann Simulations on a GPU: An optimization approach using C++ AMP, 2012.

[11] P. D. Arumuga, V. S. K. Gundavarapu, and A. K. Dass, "Lattice Boltzmann simulation of flow over a circular cylinder at moderate Reynolds numbers," Themal Science, vol. 18.4, pp. 1235-1246, 2014.

[12] Q. Zuo, and X. He, "On pressure and velocity boundary conditions for the lattice Boltzmann BGK model,” Physics of Fluids, vol. 9.6, pp. 1591-1598, 1997.

[13] Y. B. Bao, and J. Meskas, "Lattice Boltzmann method for fluid simulations," Couhrant institute of Mathematical Sciences, 2011.

[14] G. O. Brown, "The history of Darcy-Weisbach equation for pipe flow resistance," Enviormental and Water Resources History, vol. 38.7, pp. 34-43, 2002.

[15] V. Dyke, Milton, and M. V. Dyke, An album of fluid motion, 1982.

[16] C. H. K. Williamson, "Vortex dynamics in the cylinder wake," Annula review of fluid mechanics, vol. 28.1, pp. 477-539, 1996.

About Author (s)

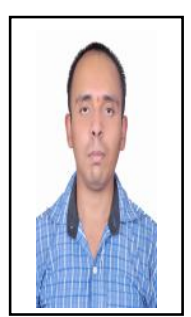

Alankar Agarwal

PhD Scholar

Department of Mechanical Engineering Indian Institute of Technology Jodhpur

Rajasthan-India 